\title{
Effects of Exercise Dose and Detraining Duration on Mobility at Late Midlife: A Randomized Clinical Trial
}

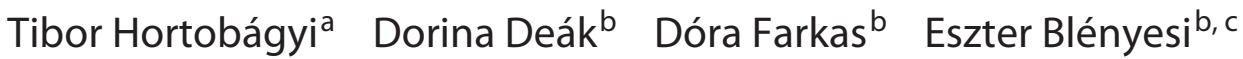 \\ Katalin Török ${ }^{b}$ Urs Granacher ${ }^{d}$ József Tollár ${ }^{b, c}$ \\ ${ }^{a}$ Center for Human Movement Sciences, University Medical Center Groningen, Groningen, The Netherlands; \\ bSomogy County Móricz Kaposi Teaching Hospital, Kaposvár, Hungary; 'Doctoral School of Health Sciences, Faculty \\ of Health Sciences, University of Pécs, Pécs, Hungary; 'Division of Training and Movement Sciences, Faculty of \\ Human Sciences, University of Potsdam, Potsdam, Germany
}

\author{
Keywords \\ Exercise $\cdot$ Dose response $\cdot$ Walking capacity $\cdot$ Cognitive \\ function
}

\begin{abstract}
Background: Office workers near retirement tend to be sedentary and can be prone to mobility limitations and diseases. We examined the dose effects of exergaming volume and duration of detraining on motor and cognitive function in office workers at late midlife to reduce sedentariness and mobility limitations. Methods: In an assessor-blinded randomized trial, 160 workers aged 55-65 years performed physically active video games in a nonimmersive form of virtual reality (exergaming) in small, supervised groups for $1 \mathrm{~h}$, $1 \times, 2 \times$, or $3 \times /$ week for 8 weeks followed by detraining for 8 and 16 weeks. Exergaming comprises high-intensity, fullbody sensorimotor coordination, balance, endurance, and strengthening exercises. The primary outcome was the 6-minute walk test (6MWT), and secondary outcomes were body mass, self-reported physical activity, sleep quality, Berg Balance Scale, Short Physical Performance Battery, fast gait speed, dynamic balance, heart rate recovery after step test, and 6 cognitive tests. Results: The 3 groups were not different in any of the outcomes at baseline (all $p>0.05$ ). The out-
\end{abstract}

comes were stable and had acceptable reliability (intraclass correlation coefficients $\geq 0.334$ ) over an 8 -week control period. Training produced an inverted $U$-shaped dose response of no $(1 \times)$, most $(2 x)$, and medium ( $3 \times$ /week) effects of exergaming volume in most motor and selected cognitive outcomes. The distance walked in the 6MWT (primary outcome) increased most (94 m, 19\%, $p<0.05)$, medium $(57 \mathrm{~m}, 12 \%$, $p<0.05)$, and least $(4 \mathrm{~m}, 1 \%)$ after exergaming $2 \times, 3 \times$, or $0 \times$ (control) (all different $p<0.05)$. The highest responders tended to retain the exercise effects over 8 weeks of detraining, independent of training volume. This maintenance effect was less consistent after 16 weeks of detraining. Conclusion: Less was more during training and lasted longer after detraining. A medium dose volume of exergaming produced the largest clinically meaningful improvements in mobility and selected cognitive tests in 60-year-old office workers with mild mobility limitations and intact cognition.

\section{(c) 2021 The Author(s)}

Published by S. Karger AG, Basel

\section{Introduction}

Physical inactivity is the fourth leading risk factor for mortality, causing $6 \%$ of deaths globally [1]. Office workers near retirement tend to be sedentary and can be prone karger@karger.com www.karger.com/ger

Karger $\stackrel{\text { ' }}{5}$

GOPEN ACCESS
(C) 2021 The Author(s)

Published by S. Karger AG, Basel

This is an Open Access article licensed under the Creative Commons Attribution-NonCommercial-4.0 International License (CC BY-NC) (http://www.karger.com/Services/OpenAccessLicense), applicable to the online version of the article only. Usage and distribution for commercial purposes requires written permission.
Tibor Hortobágyi

Center for Human Movement Sciences, University of Groningen Medical Center A. Deusinglaan 1

NL-9700 AD Groningen (The Netherlands)

t.hortobagyi@umcg.nl 
to mobility limitations and/or diseases. Office and call center workers' daily step counts are the lowest among all workers and have the greatest sitting time and least time spent on light physical activity (PA) during wakeful hours and at work [2]. Movement interventions and behavioral nudges in the office can increase in-office standing time and PA, but such changes often do not exceed $30 \mathrm{~min}$ per day, and behavioral improvements diminish over time in and away from the office, minimizing any lasting benefits for mobility, cognition, and health.

The World Health Organization (WHO) recommends that adults perform $75 \mathrm{~min}$ of vigorous- and/or $150 \mathrm{~min}$ of moderate-intensity PA per week to reduce risks for noncommunicable conditions such as cardiovascular disease, cancer, falls, and dementia [1]. The dosing of exercise duration and frequency is, however, unclear from the guidelines and can vary according to age, disease, outcome (mortality, health, and fitness [e.g., muscle strength and balance]), and motor and cognitive status at baseline. Large epidemiological studies suggest that a minimum of 150-300 min of moderate- or 75-150 min of vigorousintensity PA was associated with substantial increase in longevity benefits, which can further increase when the duration of total weekly PA is up to $450-750 \mathrm{~min} /$ week $[3,4]$. However, there is also evidence suggesting that if participants are in a physically deconditioned, sedentary state, being active less than the recommended volume may already have health-promoting effects [5] and sets people on a stable track of healthy aging [6] independent of the type of PA [7]. It is thus conceivable that even $1 \times$ per week, that is, low-volume exercise, could produce favorable effect, and $3 \times$ per week, that is, high volume of vigorous exercise, could produce a ceiling if not an overtraining effect in certain measures. We thus hypothesized that a medium volume (duration) of exercise may produce the largest effects on mobility and cognitive outcomes in office workers at late midlife.

For logistical and adherence reasons, many exercise research interventions are designed to last for a few months. Even if individuals exercise for a prolonged period, vacation, illness, or moving can interrupt the exercise regimen. Detraining, that is, the withdrawal of the exercise stimulus following a period of exercise training, is a contentious and poorly understood phenomenon, and the cumulative effects of detraining on mobility and cognition in adults at late midlife have rarely been examined. Indeed, there is evidence for lasting effects of various exercise protocols on functional outcomes and in a limited number of studies on measures of cognition in older adults $[8,9]$. However, there is an equal number of studies showing no maintenance of the exercise-induced functional and cognitive gains after detraining [10-12]. The common element emerging from these conflicting data that gives rise to our working hypothesis is that perhaps the lasting effects of exercise are actually not related to exercise parameters (i.e., intensity, volume, and frequency) but are instead related to the magnitude of gains in a given outcome. Based on the conflicting data, we tentatively hypothesize that retention of exercise-induced benefits following detraining is related to whether or not someone responds to the exercise stimulus and not to the volume of exercise performed.

Taken together, the purpose of this single-blind, randomized trial was to determine the effects of exercise volume and detraining duration on mobility and cognitive outcomes in office workers at late midlife. Because of evidence suggesting strong effects on walking capacity and cognitive function $[13,14]$, we used exergaming as an exercise stimulus that was also highly effective in patient groups, producing long-lasting effects following detraining [15].

\section{Methods}

\section{Participants and Design}

Full-time, public sector office workers $(n=160,58 \%$ female) participated in the study. Of the 345 employees, 160 were enrolled in an institution-mandated periodic health screening in the hospital where they received information about the study (online Appendix 1; see www.karger.com/doi/10.1159/000513505 for all online suppl. material). Volunteers responding to the call subsequently filled in medical and health questionnaires. The inclusion criteria were age 55-65 years, male or female gender, and a commitment to the 28-week-long program according to randomization. The exclusion criteria were Mini-Mental State Examination (MMSE) score $<20$, severe cardiac disease, uncontrolled diabetes, uncontrolled hypertension, $\mathrm{BMI}>30 \mathrm{~kg} \cdot \mathrm{m}^{-2}$, stroke or heart attack $<1$ year before, traumatic brain injury, seizure disorder, Parkinson's disease, ongoing orthopedic surgeries, pacemaker, hemophilia, current cancer, current severe cardiopulmonary conditions, use of steroids or opioids for pain, walking aids, or participation in an exercise program. A hospital physician examined all participants and decided about inclusion. The University Hospital's Ethics Committee approved the protocol and the informed consent, which each participant signed (IKEB0008/2018). The study is in agreement with the latest version of the Declaration of Helsinki.

Online Appendix 1 shows the design of the 3 -arm, single-blind, randomized clinical trial. A physical therapist not involved in the trial performed the concealed randomization of participants. $\mathrm{He}$ drew a colored ribbon from a covered box and attached one ribbon to each participant folder, designating the participant's group assignment. Group 1 (G1, $n=53,47 \%$ F) completed an initial 8-week control period to assess reliability of the outcome measures followed by an 8 -week-long exercise intervention, concluding with one 8-week detraining period. Group 2 (G2, $n=53,32 \% \mathrm{~F})$ and 
group 3 (G3, $n=54,48 \% \mathrm{~F}$ ) exercised for 8 weeks and completed two 8 -week-long detraining periods. There were 4 assessments: at baseline (test 1), after 8 weeks of control (G1) or exercise (G2 and G3, test 2), 8 weeks of training (G1) or detraining (G2 and G3) (test 3 ), and 8 weeks of additional detraining (test 4). The order of the fitness tests was standardized among participants and testing sessions. Two physical therapists (PTs) and an assistant administering the tests were masked to group assignments and experimental phase (control, exercise, and detraining).

\section{Interventions}

Exercise was administered 1 (G1), 2 (G2), or 3 (G3) times per week for $1 \mathrm{~h}$ in the hospital PT gym after work on weekdays in groups of 8-10 participants in fall 2019. The warm-up program consisted of stationary cycling for $10 \mathrm{~min}$ at $1-2 \mathrm{~kg}$ resistance. Exergaming consisted of physically active video games in a nonimmersive form of virtual reality, illustrated previously by videos [16]. Games included Xbox 360 modules, 10 min each: (1) Reflex Ridge trains reflex responses to visual stimuli; (2) Space Pop trains spatial orientation through target reaching with arms, legs, and whole body, and (3) Just Dance prompts users to generate and combine movement sequences with a strong demand on the aerobic system. Exergaming was designed to improve walking ability, gait stability, turning, postural control, and static and dynamic balance. While in the present study we did not measure heart rate during exergaming, in previous studies using similar interventions, the heart reached $80 \%$ of the age-predicted maximum, implying a high aerobic training stimulus $[17,18]$.

In addition to exergaming, strengthening exercises included plank positions for a total of $10 \mathrm{~min}$. Cooldown consisted of stretching and breathing exercises. Two PTs and an assistant administered the interventions but none of the tests. For the control period (G1) and for the detraining periods (G1, G2, and G3), participants were instructed to continue their habitual activities without changing their diet and exercise habits.

\section{Primary Outcome}

Because exercise duration was the dosing factor in the intervention, which was delivered at a high intensity (rate of perceived exertion can reach $\sim 16$ of $20[17,18]$ ), we set the 6 -minute walk test $(6 \mathrm{MWT})$ as the primary outcome. The $6 \mathrm{MWT}$ is a reliable and valid measure of walking capacity that is sensitive to change [19]. The clinically meaningful large change is $50 \mathrm{~m}$ for the $6 \mathrm{MWT}$ in mobility-limited older adults [20] but near $35 \mathrm{~m}$ for healthier and younger adults because exercise training increased 6MWT distance by an average of $36 \mathrm{~m}$ in 9 studies of healthy older adults [21].

\section{Secondary Outcomes}

PA was measured with the International Physical Activity Questionnaire (IPAQ) self-administered, short form, which has acceptable measurement properties for monitoring levels of PA among 18- to 65-year-old adults in diverse settings. Sleep quality and quantity was self-assessed with the Pittsburgh Sleep Quality Index (PSQI) that has good psychometric properties.

Because the intervention was multimodal (e.g., balance, coordination, agility, reaction time, and endurance), we also assessed mobility with a composite measure, the Short Physical Performance Battery, SPPB [22], which is a reliable and valid test of standing balance, habitual walking speed, and leg strength and is sensitive to change [20]. The Berg Balance Scale (BBS) was used to assess static balance and fall risk [23]. Dynamic balance during walking was assessed on 4-, 8-, and 12-cm-wide, 4-m-long, and 2-cm-high wooden beams [24]. Participants performed one familiarization and one measurement trial on each beam barefoot. The instruction was "Walk the entire length of the beam at your habitual speed with arms free. The trial ends when you step off." The distance, number of steps, and time to complete the trial were determined and average velocity computed, by digitizing foot markers in video recordings. Moreover, fast walking speed was measured in 3 trials over $10 \mathrm{~m}$, including acceleration and deceleration. Cardiovascular endurance was assessed with the 3-min-long box step test, and recovery heart rate during minute 4 was used to assess change in fitness [25].

We used MMSE to measure cognitive impairment. We measured motor speed and attention with the Digit Symbol Substitution Test (DSST) and inhibition of cognitive interference with the Stroop color-word test. Memory span and working memory were measured with the Digit Span (DS) and Visual Memory Span (VMS) Forward and Backward.

\section{Statistical Analyses}

Using $\mathrm{G}^{*}$ Power (version 3.1.9.2.), we estimated the number of participants needed for a significant group (G1, G2, and G3) by time (pre and post) interaction for the primary outcome. A priori power analysis revealed the need for 44 participants per group with a clinically meaningful increase of $50 \mathrm{~m}$ in the 6MWT, the primary outcome [20], producing a medium effect of $0.5(\alpha=0.05$; power $=$ $1-\beta$ [power] of 0.8 ). Because the reliability analysis showed stability of the measures upon a retest after 8 weeks $(n=53)$ and there were no differences between the 3 groups in the outcome measures at baseline, the main analysis was a 1-way ANOVA on post minus pre (delta) scores for each outcome measure. Continuous variables were normally distributed based on the Shapiro-Wilk test. Categorical variables were analyzed with a Kruskal-Wallis test. A significant effect, characterized by $\mathrm{p \eta}^{2}$ effect size, was interpreted as a group by time interaction and was followed by a Tukey's post hoc or a Mann-Whitney test to determine the means that differed from one another. Within-group changes were further quantified by computing Cohen's $d$. The Holm method was used to correct for family-wise error. The level of significance was set at $p<0.05$.

\section{Results}

\section{Baseline Characteristics}

Enrolled clerical employees were legal staff (28\%), office assistants (22\%), economists (9\%), police (9\%), service staff $(8 \%)$, physicians $(8 \%)$, engineers $(5 \%)$, teachers (5\%), and business and other consultants (3\%). Of the 160 enrolled, 53\% had a treated illness: hypertension $(n=29)$, diabetes (21), past stroke (11), benign paroxysmal positional vertigo (11), phobic postural vertigo (5), or heart attack, ulcerative colitis, and Crohn's disease ( $n=1$ each). The repeat tests 8 weeks apart revealed acceptable reliability for all variables, and the 3 groups were not different in outcomes at baseline (all $p>0.05$ ) (online suppl. Appendices 2, 3). 
Table 1. Effects of exercise volume on outcome measures

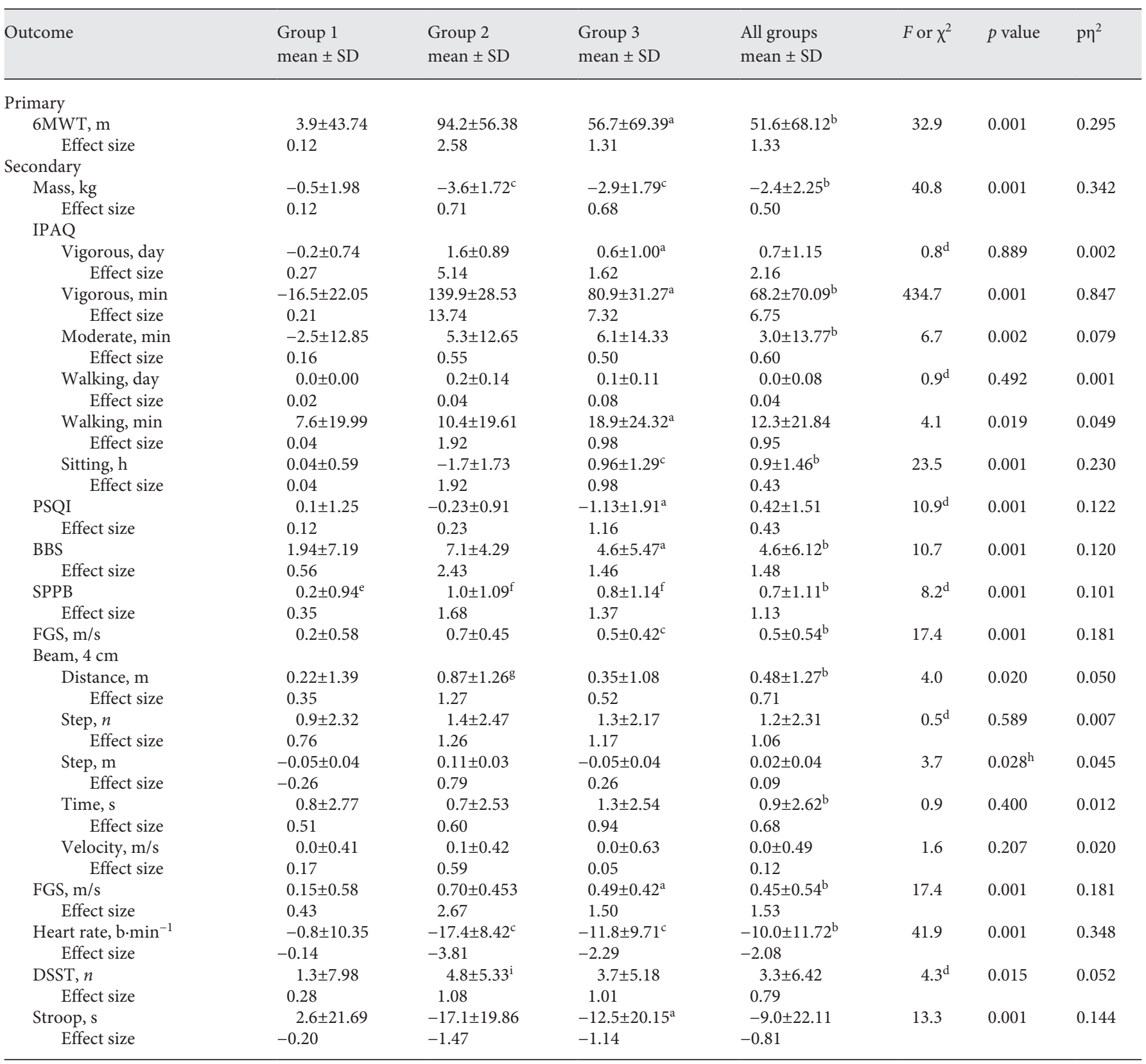

Values are post minus pre delta scores in absolute units. Groups 1,2 , and 3 exercise $1 \times, 2 \times$, and $3 \times$ per week for $1 \mathrm{~h}$ each. $F$, Fisher's test. $p$, probability $<0.05$ denotes a group by time interaction based on the change scores. $\chi^{2}$, Kruskal-Wallis test. $\eta^{2}$, partial eta squared; small effect: $0.02-0.12$, medium: $0.13-0.25$, and large $\geq 0.26$. Cohen's effect size: small $\leq 0.50$; medium $0.50-0.79$, and large $\geq 0.80$. $6 \mathrm{MWT}, 6$-minute walk test; IPAQ, International Physical Activity Questionnaire; vigorous, day, the number of days participants reported conducting vigorous PA; vigorous, min, daily minutes participants reported conducting vigorous PA; moderate, min, daily minutes participants reported conducting vigorous PA; walking, day, number of days per week participants reported to walk; walking min, number of minutes participants reported to walk in a day; sitting, h, the number of hours participants reported to sit in a day; PSQI, Pittsburgh Sleep Quality Index; BBS, Berg Balance Scale; SPPB, Short Physical Performance Battery; FGS, fast gait speed; heart rate, during 1 min after 3 min of stepping; DSST, number of correct responses in the Digit Symbol Substitution Test; Stroop, completion time of the Stroop color-word test; PA, physical activity. ${ }^{\text {a }}$ All 3 means differ from one another, $p<0.05 .{ }^{\mathrm{b}}$ Significantly change relative to a change of zero, $p<0.05 .{ }^{\mathrm{c}}$ Greater changes in G2 and G3 than in G1. ${ }^{\mathrm{d}}$ Kruskal-Wallis test. ${ }^{\mathrm{e}} \mathrm{G} 1$ differs from G3, $p<0.05 .{ }^{\mathrm{f}}$ Greater change than in G1. ${ }^{\mathrm{g}} \mathrm{G} 2$ differs from other 2 groups, $p<0.05 .{ }^{\mathrm{h}}$ Did not survive Holm's correction for family-wise error. ${ }^{i}$ G2 differs from G1 and G3. 
Table 2. Effects of 8 weeks of detraining on the outcomes

\begin{tabular}{|c|c|c|c|c|c|c|c|}
\hline Outcome & $\begin{array}{l}\text { Group } 1 \\
\text { mean } \pm \text { SD }\end{array}$ & $\begin{array}{l}\text { Group } 2 \\
\text { mean } \pm S D\end{array}$ & $\begin{array}{l}\text { Group } 3 \\
\text { mean } \pm \text { SD }\end{array}$ & $\begin{array}{l}\text { All groups } \\
\text { mean } \pm S D\end{array}$ & $F$ or $\chi^{2}$ & $p$ value & $\mathrm{p} \eta^{2}$ \\
\hline \multicolumn{8}{|l|}{ Primary } \\
\hline 6MWT, m & $-1.5 \pm 64.2$ & $83.8 \pm 54.15$ & $42.9 \pm 78.47^{\mathrm{a}}$ & $41.8 \pm 74.65^{\mathrm{b}}$ & 21.8 & 0.001 & 0.218 \\
\hline Effect size & -0.04 & 2.71 & 1.05 & 1.25 & & & \\
\hline \multicolumn{8}{|l|}{ Secondary } \\
\hline Mass, kg & $-0.6 \pm 1.97$ & $-3.6 \pm 1.97$ & $-2.8 \pm 2.17^{\mathrm{c}}$ & $-2.4 \pm 2.39^{b}$ & 30.3 & 0.001 & 0.278 \\
\hline Effect size & -0.14 & -0.71 & -0.65 & -0.50 & & & \\
\hline \multicolumn{8}{|l|}{ IPAQ } \\
\hline Vigorous, min & $-12.0 \pm 17.16$ & $80.7 \pm 47.94$ & $56.9 \pm 44.55^{\mathrm{a}}$ & $41.9 \pm 55.26^{\mathrm{b}}$ & 80.3 & 0.001 & 0.505 \\
\hline Effect size & -0.58 & 3.00 & 2.62 & 1.70 & & & \\
\hline Walking, min & $-0.8 \pm 5.49$ & $11.7 \pm 26.22$ & $10.4 \pm 24.87^{\mathrm{c}}$ & $7.1 \pm 21.73$ & 5.6 & 0.005 & 0.066 \\
\hline Effect size & -0.05 & 0.78 & 0.68 & 0.47 & & & \\
\hline Sitting, h & $-0.3 \pm 0.73$ & $-1.6 \pm 1.69$ & $-1.1 \pm 1.79^{c}$ & $-1.0 \pm 1.57^{\mathrm{b}}$ & 10.0 & 0.001 & 0.113 \\
\hline Effect size & 0.33 & 1.77 & 1.26 & 1.12 & & & \\
\hline PSQI & $0.0 \pm 0.00$ & $0.2 \pm 1.36$ & $-1.0 \pm 1.81^{\mathrm{a}}$ & $-0.3 \pm 1.39$ & $12.6^{\mathrm{d}}$ & 0.001 & 0.134 \\
\hline Effect size & 0.00 & 0.19 & 1.04 & 0.28 & & & \\
\hline $\mathrm{BBS}$ & $1.7 \pm 8.99$ & $6.8 \pm 5.62$ & $4.8 \pm 6.57^{\mathrm{c}}$ & $4.4 \pm 7.44^{\mathrm{b}}$ & 6.8 & 0.001 & 0.080 \\
\hline Effect size & 0.43 & 2.23 & 1.54 & 1.40 & & & \\
\hline SPPB & $0.1 \pm 0.88$ & $0.9 \pm 1.13$ & $0.6 \pm 1.02^{\mathrm{c}}$ & $0.5 \pm 1.06^{\mathrm{b}}$ & $7.9^{\mathrm{d}}$ & 0.001 & 0.092 \\
\hline Effect size & 0.17 & 1.38 & 1.04 & 0.86 & & & \\
\hline $\mathrm{FGS}, \mathrm{m} / \mathrm{s}$ & $0.0 \pm 0.53$ & $0.5 \pm 0.68$ & $0.4 \pm 0.75^{\mathrm{c}}$ & $0.31 \pm 0.69$ & 8.0 & 0.001 & 0.092 \\
\hline Effect size & 0.09 & 1.51 & 0.96 & 0.85 & & & \\
\hline \multicolumn{8}{|l|}{ Beam, $4 \mathrm{~cm}$} \\
\hline Distance, $\mathrm{m}$ & $0.0 \pm 1.67$ & $0.7 \pm 1.28$ & $0.3 \pm 1.41$ & $0.3 \pm 1.48^{\mathrm{b}}$ & 2.8 & 0.066 & 0.034 \\
\hline Effect size & 0.00 & 1.03 & 0.40 & 0.49 & & & \\
\hline Step, m & $-0.16 \pm 0.43$ & $0.16 \pm 0.44^{\mathrm{g}}$ & $-0.09 \pm 0.42$ & $-0.03 \pm 0.44$ & 8.0 & 0.001 & 0.093 \\
\hline Effect size & -0.75 & 0.77 & -0.42 & 0.13 & & & \\
\hline Heart rate, $\mathrm{b} \cdot \mathrm{min}^{-1}$ & $0.3 \pm 9.62$ & $-15.5 \pm 9.56$ & $-9.6 \pm 9.10^{\mathrm{a}}$ & $-8.4 \pm 11.42^{\mathrm{b}}$ & 38.1 & 0.001 & 0.32 \\
\hline Effect size & 0.04 & -3.35 & -1.75 & 1.69 & & & \\
\hline DSST, $n$ & $0.2 \pm 9.19$ & $-0.2 \pm 8.49$ & $2.6 \pm 6.74$ & $1.0 \pm 8.28$ & $2.1^{\mathrm{d}}$ & 0.146 & 0.020 \\
\hline Effect size & 0.04 & -0.04 & 0.74 & 0.24 & & & \\
\hline Stroop, s & $4.2 \pm 20.57$ & $-11.0 \pm 16.58$ & $-5.8 \pm 20.66^{c}$ & $-4.2 \pm 20.9^{b}$ & 7.8 & 0.001 & 0.090 \\
\hline Effect size & 0.35 & -0.89 & -0.48 & -0.34 & & & \\
\hline
\end{tabular}

Values are post- 8 weeks of detraining minus pretraining delta scores in absolute units. Variables that showed no training effects in Table 2 are omitted from detraining analyses. Groups 1, 2, and 3 exercise 1 h each $1 \times, 2 \times$, or $3 \times$ per week for 8 weeks. F, Fisher's test. $p$, probability $<0.05$ denotes a group by time interaction based on the change scores. $\chi^{2}$, Kruskal-Wallis test. p $\eta^{2}$, partial eta squared; small effect: $0.02-0.12$, medium: $0.13-0.25$, and large $\geq 0.26$. Cohen's effect size: small $\leq 0.50$, medium $0.50-0.79$, and large $\geq 0.80$. $6 \mathrm{MWT}$, 6-minute walk test; IPAQ, International Physical Activity Questionnaire; vigorous, day, the number of days participants reported conducting vigorous PA; vigorous, min, daily minutes participants reported conducting vigorous PA; moderate, min, daily minutes participants reported conducting vigorous PA; walking, day, number of days per week participants reported to walk; walking, min, number of minutes participants reported to walk in a day; sitting, h, number of hours participants reported to sit in a day; PSQI, Pittsburgh Sleep Quality Index; BBS, Berg Balance Scale; SPPB, Short Physical Performance Battery; FGS, fast gait speed; heart rate, during 1 min after 3 min of stepping; DSST, number of correct responses in the Digit Symbol Substitution Test; Stroop, completion time of the Stroop colorword test; PA, physical activity. ${ }^{\text {a }}$ All 3 means differ from one another, $p<0.05$. ${ }^{\mathrm{b}}$ Significant change relative to a change of zero, $p<0.05$. ${ }^{c}$ Greater changes in G2 and G3 than in G1. ${ }^{\mathrm{d}}$ Kruskal-Wallis test.

\section{Effects of 8 Weeks of Intervention}

Table 1 shows the training effects, which tended to be the greatest in G2 followed by G3 and G1. The intervention improved the distance walked in the primary outcome (6MWT) by $\sim 52 \mathrm{~m}$ or $11 \%(p<0.05)$. The largest increase occurred in G2 (94 m, 19\%) followed by G3 (57 $\mathrm{m}, 12 \%)$ and $\mathrm{G} 1(4 \mathrm{~m}, 1 \%)$ (all different $p<0.05)$.

Body mass decreased by $3.1 \%$ in the 3 groups combined $(p<0.05)$. The weight loss was similar in G2 $(4.6 \%)$ and G3 $(3.8 \%)$ but greater than in G1 (0.6\%). The number 
Table 3. Effects of 8 and 16 weeks of detraining on the outcomes

\begin{tabular}{|c|c|c|c|c|c|c|c|c|c|}
\hline \multirow[t]{2}{*}{ Outcome } & \multicolumn{3}{|l|}{8 weeks } & \multicolumn{6}{|l|}{16 weeks } \\
\hline & $\begin{array}{l}\text { group } 2 \\
\text { mean } \pm \mathrm{SD}\end{array}$ & $\begin{array}{l}\text { group } 3 \\
\text { mean } \pm \mathrm{SD}\end{array}$ & $\begin{array}{l}\text { both } \\
\text { mean } \pm S D\end{array}$ & $\begin{array}{l}\text { group } 2 \\
\text { mean } \pm \mathrm{SD}\end{array}$ & $\begin{array}{l}\text { group } 3 \\
\text { mean } \pm S D\end{array}$ & $\begin{array}{l}\text { both } \\
\text { mean } \pm S D\end{array}$ & $\begin{array}{l}\mathrm{G} \\
\mathrm{p} \eta^{2}\end{array}$ & $\begin{array}{l}\mathrm{T} \\
\mathrm{p} \eta^{2}\end{array}$ & $\begin{array}{l}\mathrm{G} \times \mathrm{T} \\
\mathrm{p} \eta^{2}\end{array}$ \\
\hline \multicolumn{10}{|l|}{ Primary } \\
\hline 6MWT, m & $83.8 \pm 54.15$ & $42.9 \pm 78.47$ & $63.2 \pm 70.26$ & $75.5 \pm 73.92$ & $39.6 \pm 71.58$ & $57.4 \pm 74.39$ & $0.090^{\mathrm{a}}$ & 0.010 & 0.001 \\
\hline Effect size & 2.71 & 1.25 & 1.98 & 2.06 & 0.91 & 1.49 & & & \\
\hline \multicolumn{10}{|l|}{ Secondary } \\
\hline $\begin{array}{l}\text { Mass, kg } \\
\text { Effect size }\end{array}$ & $\begin{array}{l}-3.6 \pm 1.97 \\
-0.71\end{array}$ & $\begin{array}{l}-2.8 \pm 2.17 \\
-0.65\end{array}$ & $\begin{array}{l}-3.2 \pm 2.10 \\
-0.68\end{array}$ & $\begin{array}{l}-3.5 \pm 1.89 \\
-0.69\end{array}$ & $\begin{array}{l}-2.5 \pm 2.39 \\
-0.58\end{array}$ & $\begin{array}{l}-3.0 \pm 2.21 \\
-0.64\end{array}$ & $0.050^{\mathrm{a}}$ & 0.035 & 0.017 \\
\hline \multicolumn{10}{|l|}{ IPAQ } \\
\hline Vigorous, min & $80.7 \pm 47.94$ & $56.9 \pm 44.55$ & $68.6 \pm 46.57$ & $65.4 \pm 49.01$ & $-7.8 \pm 16.53$ & $28.5 \pm 32.99$ & $0.357^{\mathrm{a}}$ & $0.383^{\mathrm{b}}$ & $0.191^{\mathrm{c}}$ \\
\hline Effect size & 3.00 & 2.62 & 2.81 & 2.73 & -0.36 & -1.18 & & & \\
\hline Walking, min & $11.7 \pm 26.22$ & $10.4 \pm 24.87$ & $10.8 \pm 25.52$ & $9.2 \pm 28.69$ & $0.0 \pm 0.00$ & $4.6 \pm 14.34$ & 0.023 & 0.047 & 0.022 \\
\hline Effects size & 0.78 & 0.68 & 0.73 & 0.65 & 0.00 & 0.32 & & & \\
\hline Sitting, $\mathrm{h}$ & $-1.6 \pm 1.69$ & $-1.1 \pm 1.79$ & $-1.3 \pm 1.75$ & $-1.5 \pm 1.90$ & $-0.8 \pm 1.52$ & $-1.1 \pm 1.75$ & $0.037^{\mathrm{a}}$ & 0.024 & 0.014 \\
\hline Effect size & -1.77 & -1.26 & -1.51 & -1.69 & -0.78 & -0.93 & & & \\
\hline $\mathrm{PSI}^{\mathrm{d}}$ & $0.2 \pm 1.36$ & $-1.0 \pm 1.81$ & $-0.4 \pm 1.69$ & $0.4 \pm 1.37$ & $-0.6 \pm 2.09$ & $-0.1 \pm 1.83$ & $0.121^{\mathrm{a}}$ & $0.037^{\mathrm{b}}$ & 0.002 \\
\hline Effect size & 0.19 & -1.04 & -0.42 & 0.42 & -0.61 & -0.09 & & & \\
\hline $\mathrm{BBS}$ & $6.8 \pm 5.56$ & $4.8 \pm 6.57$ & $5.8 \pm 6.10$ & $5.9 \pm 6.68$ & $4.4 \pm 7.05$ & $5.1 \pm 6.86$ & 0.002 & 0.011 & 0.002 \\
\hline Effect size & 2.23 & 1.54 & 1.88 & 1.82 & 1.27 & 1.17 & & & \\
\hline $\mathrm{SPPB}^{\mathrm{d}}$ & $0.9 \pm 1.13$ & $0.6 \pm 1.02$ & $0.7 \pm 1.06$ & $0.8 \pm 1.08$ & $0.6 \pm 1.19$ & $0.7 \pm 1.13$ & 0.013 & 0.001 & 0.000 \\
\hline Effect size & 1.38 & 1.04 & 1.21 & 1.39 & 1.04 & 1.22 & & & \\
\hline FGS & $0.5 \pm 0.68$ & $0.4 \pm 0.75$ & $0.4 \pm 0.71$ & $0.5 \pm 0.57$ & $0.2 \pm 0.76$ & $0.4 \pm 0.66$ & 0.002 & 0.021 & 0.002 \\
\hline Effect size & 1.51 & 0.96 & 1.23 & 1.58 & 0.69 & 1.14 & & & \\
\hline \multicolumn{10}{|l|}{ Beam, $4 \mathrm{~cm}$} \\
\hline Distance, $\mathrm{m}$ & $0.7 \pm 1.28$ & $0.3 \pm 1.41$ & $0.5 \pm 1.35$ & $0.6 \pm 1.5$ & $0.2 \pm 1.3$ & $0.4 \pm 1.38$ & 0.029 & 0.006 & 0.002 \\
\hline Effect size & 1.03 & 0.40 & 0.73 & 0.84 & 0.23 & 0.53 & & & \\
\hline Step length & $0.16 \pm 0.44$ & $-0.09 \pm 0.42$ & $0.27 \pm 0.35$ & $0.14 \pm 0.41$ & $-0.09 \pm 0.42$ & $0.22 \pm 0.42$ & $0.101^{\mathrm{a}}$ & 0.000 & 0.000 \\
\hline Effect size & 0.77 & -0.42 & 0.17 & 0.71 & -0.44 & 0.13 & & & \\
\hline Heart rate, $b \cdot \min ^{-1}$ & $-15.5 \pm 9.56$ & $-9.6 \pm 9.10$ & $-12.5 \pm 8.96$ & $-15.5 \pm 9.65$ & $-9.9 \pm 9.10$ & $-12.7 \pm 9.37$ & $0.109^{\mathrm{a}}$ & 0.001 & 0.001 \\
\hline Effect size & -3.35 & -1.75 & -2.55 & -3.19 & -1.83 & -2.51 & & & \\
\hline DSST, $n^{\mathrm{d}}$ & $-0.2 \pm 8.49$ & $2.6 \pm 6.74$ & $1.2 \pm 7.52$ & $0.2 \pm 12.87$ & $1.33 \pm 6.56$ & $0.8 \pm 9.71$ & 0.015 & 0.002 & 0.001 \\
\hline Effect size & -0.04 & 0.74 & 0.35 & 0.03 & -0.35 & -0.20 & & & \\
\hline Stroop, s & $-11.0 \pm 16.58$ & $-5.8 \pm 20.66$ & $-8.4 \pm 20.00$ & $-11.9 \pm 17.00$ & $-4.7 \pm 20.66$ & $-8.3 \pm 18.84$ & 0.032 & 0.000 & 0.001 \\
\hline Effect size & -0.89 & -0.48 & -0.69 & -1.06 & -0.43 & -0.74 & & & \\
\hline
\end{tabular}

Values are after 8 and 16 weeks of detraining minus pretraining delta scores in absolute units. Variables that showed no training effects in Table 2 or detraining effects are omitted from this table. Groups 2 and 3 exercised 1 h each $2 \times$ and $3 \times$ per week for 8 weeks. G, group main effect. T, time main effect. $\mathrm{G} \times \mathrm{T}$, group by time interaction. $\eta^{2}$, partial eta squared; small effect: $0.02-0.12$, medium effect: $0.13-0.25$, and large effect: $\geq 0.26$. $\eta^{2}$, for PSI, SPPB, and DSST. Cohen's effect size: small $\leq 0.50$, medium 0.50-0.79, and large $\geq 0.80$. 6MWT, 6-minute walk test; IPAQ, International Physical Activity Questionnaire; vigorous, min, daily minutes participants reported conducting vigorous PA; walking min, number of minutes participants reported to walk in a day; sitting, h, number of hours participants reported to sit in a day; PSQI, Pittsburgh Sleep Quality Index; BBS, Berg Balance Scale; SPPB, Short Physical Performance Battery; FGS, fast gait speed; heart rate, during $1 \mathrm{~min}$ after $3 \mathrm{~min}$ of stepping; DSST, number of correct responses in the Digit Symbol Substitution Test; Stroop, completion time of the Stroop color-word test; PA, physical activity. ${ }^{\text {a }}$ Group main effect $(p<0.05)$. ${ }^{\text {b }}$ Time main effect $(p<0.05) .{ }^{\mathrm{c}}$ Group by time interaction $(p<0.05) .{ }^{\mathrm{d}}$ Kruskal-Wallis test.

of days participants reported to perform vigorous PA did not change, but the number of minutes of vigorous and moderate PA increased overall by $\sim 70 \mathrm{~min}$ in the 3 groups $(p<0.05)$. The largest changes occurred in G2 $(\sim 2 \mathrm{~h})$, followed by G3 (90 min) and G1 ( $10 \mathrm{~min})$. The number of minutes participants reported they walked in a day in- creased by $\sim 9 \mathrm{~min}$ in $\mathrm{G} 3,66 \mathrm{~min}$ in $\mathrm{G} 1$, and $57 \mathrm{~min}$ in $\mathrm{G} 2$. Daily sitting time $(8.5 \mathrm{~h}$ ) decreased by $0.9 \mathrm{~h}$ in the 3 groups $(p<0.05)$, by $1.7 \mathrm{~h}$, the most, in G2, compared with the $\sim 1 \mathrm{~h}$ reduction in G3 without changes in G1. Sleep quality decreased the most in G3 by one unit $(p<0.05)$. 


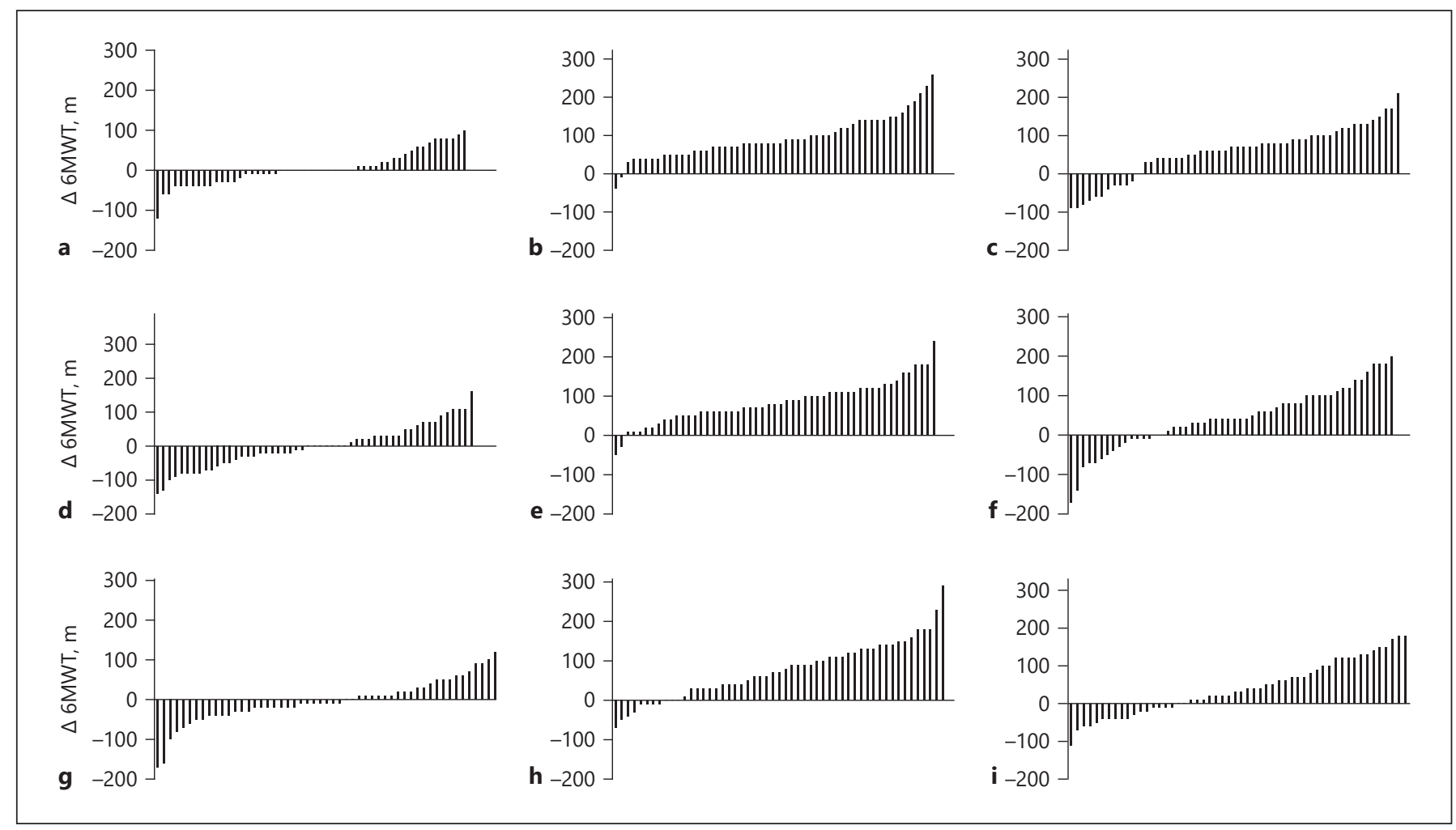

Fig. 1. Distribution of individual changes in the primary outcome, the 6-minute walk test (6MWT), after training $1 \times(\mathbf{a}, n=53$, mean: $4 \mathrm{~m}), 2 \times(\mathbf{b}, n=53,94 \mathrm{~m})$, and $3 \times(\mathbf{c}, n=54,57 \mathrm{~m})$ a week for 8 weeks. The change scores are computed as posttraining minus pretraining values. The effects of detraining for 8 weeks after training $1 \times, 2 \times$, and $3 \times$ per week (d [mean: $-2 \mathrm{~m}$ ], e [84 m], and f [43 m]). The effects of detraining for additional 8 weeks, a total of 16 weeks (h [mean: $76 \mathrm{~m}$ ] and $\mathbf{i}[40 \mathrm{~m}]$ ). The detraining change scores are computed as post-detraining minus pretest values with negative

Scores in BBS improved by $13 \%$ in the 3 groups $(p<$ $0.05)$, the most in G2 (20\%) compared with G3 (13\%). These changes exceeded the 2 points or $7 \%$ increase in G1. SPPB improved overall by 0.7 points or $9 \%$ : the 13 and $11 \%$ improvements in G2 and G3 were similar, and these changes exceeded the small changes in G1. Fast gait speed improved overall by $0.5 \mathrm{~m} / \mathrm{s}$ or $26 \%(p<0.05)$, with similar increases of $39 \%(0.69 \mathrm{~m} / \mathrm{s})$ and $28 \%(0.49 \mathrm{~m} / \mathrm{s})$ in G2 and G3, exceeding the $11 \%(0.2 \mathrm{~m} / \mathrm{s})$ increase $(p>0.05)$ in G1. There was a ceiling effect for beam walking distance on the 12- and 8-cm-wide beams (no further data are shown for these conditions). On the $4-\mathrm{cm}$ beam, distance walked increased overall by $\sim 0.5 \mathrm{~m}(p<0.05)$. The improvements in G2 $(0.9 \mathrm{~m}, 166 \%)$ exceeded the changes in G3 $(0.3 \mathrm{~m})$ and $\mathrm{G} 1(0.2 \mathrm{~m})$. The number of steps on the beam increased overall by 1.2 or $73 \%$. The time to complete the trials overall increased by $\sim 1.0 \mathrm{~s}$. Heart rate dur- scores denoting lower performance after detraining compared with pretraining. $\mathbf{h}$ The $76-\mathrm{m}$ mean value after detraining for 16 weeks means that performance in the $6 \mathrm{MWT}$ was still maintained $76 \mathrm{~m}$ above the baseline level. $\mathbf{g}$ (Mean: $-5 \mathrm{~m}$ ). The changes as post minus pre of an 8-week-long no-training control period. Change scores in each panel are sorted left to right in order from the largest loss to the largest gain in distance walked in $6 \mathrm{~min}$. Zero changes were edited to a value of 0.1 for illustration purposes.

ing the step test decreased by $10 \mathrm{~b} \cdot \mathrm{min}^{-1}(p<0.05)$. The decreases were more $(p<0.05)$ in G2 and G3 than in G1 $(p>0.05)$. Of the 6 cognitive tests, performance in DSST improved in the 3 groups combined by $13 \%(p<0.05)$, driven by the $17 \%$ in G2 $(p<0.05)$. The performance in the Stroop test improved by -13 and $-9 \%$ in G2 and G3 (both $p<0.05)$, more than the $4 \%$ change in G1 $(p>0.05)$.

\section{Effects of 8 and 16 Weeks of Detraining}

In general, the little training effect helped participants in G1 to minimize losses in the outcomes during 8 weeks of detraining. In most outcomes, detraining effects were similar in G2 and G3 (Tables 2, 3).

Relative to baseline performance in the 6MWT, G2 still walked the longest distance $(\sim 84 \mathrm{~m})$ compared with G3 $(\sim 43 \mathrm{~m})$ and G1 $(\sim-2 \mathrm{~m}$, all different $p<0.05)$, and G2 versus G3 still walked $\sim 38 \mathrm{~m}(d=2.88)$ longer dis- 
tance after 16 weeks of detraining. Figure 1 shows the individual responses to detraining in the 6MWT. After 8 weeks of detraining, 72,68 , and $76 \%$ of the participants in G1, G2, and G3 and, after 16 weeks of detraining, 51 and $47 \%$ in G2 and G3 had 6MWT performance above baseline.

G2 $(-5 \%)$ and G3 (-4\%) have maintained traininginduced weight loss after 8 and 16 weeks of detraining. G2 has retained the training-induced increases in vigorous PA at 8 and 16 weeks of detraining compared with G3 and G1, so that at 16 weeks, G2 reported still $\sim 1 \mathrm{~h}$ more $(d=$ 2.22 ) vigorous $P A$ which decreased below baseline in G3. Detraining did not affect the number of minutes walked per day. The difference in retention of reduced sitting time between G2 and G3 ( 0.5 h) after 8 weeks of detraining increased to $\sim 1.0 \mathrm{~h}(p<0.05, d=1.27)$. Detraining did not affect sleep quality.

Relative to baseline, the training-induced greater $(p<$ $0.05)$ benefits were retained similarly $(p>0.05)$ in G2 and G3 compared with G1 at 8 and 16 weeks of detraining in fall risk (BBS), mobility and balance (SPPB), fast walking speed, and distance walked on the 4-cm-wide beam. G2 compared with G1 and G3 made longer steps while walking on the narrow beam and retained these longer steps at 16 weeks. Recovery heart rates after the step test decreased the most in G2 compared with the other 2 groups, and these reductions were better retained in G2 than G3 at 16 weeks of detraining. Detraining for any duration did not affect performance in DSST, but G2 and G3 versus G1 retained improvements in the Stroop test better, and additional 8 weeks of detraining did not further affect the differences in this test between G2 and G3.

\section{Discussion}

According to the hypotheses, we found that less was more during training and lasted longer after detraining: medium duration dose of exergaming produced the largest clinically meaningful improvements in mobility and selected cognitive tests in 60-year-old office workers with mild mobility limitations and intact cognition.

\section{Sample Characteristics at Baseline}

Near retirement, over $50 \%$ of the 160 participants had a current or past medical condition but had normal body and fat mass. Participants' PA level met WHO guidelines, as self-reported daily walking and vigorous PA amounted to $40-50 \mathrm{~min}$ (online Appendix 2). Daily sitting time was $8.5 \mathrm{~h}$ or $\sim 60 \%$ of wakeful time, substantially lower than the 10.0-10.6 h reported for office workers in other countries [26]. These encouraging shorter daily sitting and longer walking times may be related to the rural setting of the trial that stimulates walking and the use of public transportation. While all outcomes, including IPAQ, were stable over the 8-week-long no-intervention control period (online Appendix 3), the reliability and validity of IPAQ are unclear, as some studies report $\sim 80 \%$ overestimation of PA in community-dwelling adults [27]. The SPPB score of 8.5 suggests limitations in mobility, leg strength, and balance [22]. Yet, the 534-m distance in the $6 \mathrm{MWT}$ and the $2.1-\mathrm{m} / \mathrm{s}$ fast gait speed suggest no gait impairments relative to the $529 \mathrm{~m}$ and $1.9 \mathrm{~m} / \mathrm{s}$ norms [17, $21]$. BBS scores were below 45 , indicating no fall risk. A lack of correlation among SPPB, 6MWT, and fast gait speed at baseline (data not shown) indicates that each test measures a unique element of mobility so that these measures are not redundant. Beam walking measures dynamic balance [24] and revealed ceiling effects on the 8- and 12 -cm-wide beams. However, the 4 - $\mathrm{cm}$-wide beam poses a strong challenge that all participants were still able to manage. We thus strongly recommend future studies to use this test to measure dynamic balance, as the test does not suffer from floor or ceiling effects. Processing speed, inhibition, and memory measures were normal but did not correlate with each other or motor outcomes (data not shown). In sum, despite medical history and mild mobility limitation per SPPB, participants of the present study were apparently healthy community-dwelling office workers late midlife.

\section{Training Effects}

The high-intensity exercise stimulus was designed and proved previously to be effective to reduce body mass and improve mobility, balance, and whole-body sensorimotor coordination in patients with Parkinson's disease, multiple sclerosis, and older adults with severe mobility limitations [16-18]. In the primary outcome, the 6MWT, the distance walked increased in an inverted U-shaped dose response by 4,94 , and $56 \mathrm{~m}$ in G1, G2, and G3 (Fig. 1; Table 1). The increase in G2 is nearly double of the $50 \mathrm{~m}$ clinically meaningful change [20]. A variety of exercise interventions increased 6MWT distance much less, by only $36 \mathrm{~m}$ in healthy older adults [21]. Perhaps, the fitness level of those compared with our participants was lower, accounting in part for the much larger improvements we observed. The potency of the exercise intervention in G2 and G3 is signified by the unusually large increases in walking distance notwithstanding participants' relatively high level of daily PA and low daily sitting time at baseline (online Appendix 2). 
Most secondary outcomes also revealed an inverted Ushaped dose-response pattern favoring G2 (Table 1). Decreases of up to $5 \%$ in body mass agree with weight loss reported after multimodal training in adults and can meaningfully reduce risks for cardiovascular disease even in normal-weight adults at mid-late life [28]. The pattern of changes in PA indeed raises questions about the IPAQ's validity because while participants in G2 reported the expected increases in (vigorous) PA in proportion to the $2 \times$ per week exercise, that was not the case in G3, reporting in fact less increase in PA than G2 and G1 (Table 1). The large effect-size changes in G2 followed by G3 in BBS, fast gait speed, and dynamic balance on the 4 - $\mathrm{m}$ beam provide compelling evidence for the efficacy of the highly challenging exergaming program to improve fall risk, walking capacity, and dynamic balance. Particularly relevant is the $\sim 1.0$ increase in SPPB, freeing participants from mild mobility limitation. Comparing with the $0.1 \mathrm{~m}$ change $(p>$ 0.05 ) in distance walked on the 4-cm beam in the control period (online Appendix 3), G2 walked nearly $1.0 \mathrm{~m}$ longer distance on the beams using 1.4 more steps, suggesting that participants in G2 had become more confident and comfortable facing the strong challenge and chose a strategy that kept them on the beam longer. The improvements in mobility- and balance-related outcomes are also encouraging in light of data suggesting no additional improvements in mobility with nutritional supplementation [29].

The present study is highly relevant for exercise prescription. In the context of the study, $2 \times /$ weekly exergaming seems to be the optimal training volume for improving most outcomes in community-dwelling adults at late mildlife. There are virtually no studies examining the effects of training volume in the context of the present study, but a meta-analytic review concluded that $90-120 \mathrm{~min}$ of balance training per week is the most effective in improving overall balance performance in older adults [30]. It is not clear from the present data why once a week exergaming was ineffective. We speculate that intense $3 \times$ /week exergaming might have caused overtraining because sleep quality decreased more than exercising once or twice a week. This speculation is in line with reductions in exercise and spontaneous PA in G3. An additional novel element of the high-intensity exergaming program is that not only did it improve mobility and balance-related outcomes but it did so by decreasing the cardiovascular load, as the heart rate during the step test decreased $12-17$ beats. $\mathrm{min}^{-1}$. This is an important finding because increased fitness could reduce fatigue, a condition adults report frequently at late midlife [31].

Exergaming and Detraining at Late Midlife
Motor and cognitive function declines with age in a correlated manner due to aging-related changes in brain circuitry, pathology, and molecular fidelity [32-35]. Our data strengthen the moderate evidence that exergaming can improve cognitive function in healthy adults at late midlife [13]. Of 6 cognitive tests, the exergaming-induced improvements in cognition were limited to DSST and the Stroop test, favoring $2 \times /$ weekly exergaming (Table 1 ), suggesting small but significant improvements in inhibition of cognitive interference. These results, while limited, are important because participants were office workers with normal cognition at baseline. The data, however, provide no support for the hypothesized correlated improvements in motor and cognitive function, as we found no association between individual changes in motor and cognitive scores (data not shown). Taken the training data together, we found evidence for the "less ( $2 \times$ vs. $3 \times$ per week) is more" phenomenon in the context of the present study.

\section{Detraining Effects}

We observed a dissociation between the inverted Ushaped dose effects of exergaming on the 6MWT, the primary outcome, and the ensuing detraining effects. In $\sim 72$ and $\sim 50 \%$ of the 160 participants, the training effects outlasted the training period for 8 and 16 weeks, respectively (Tables 2,3). Figure 2 shows that the detraining effects at 8 weeks were related to the magnitude of training gains but not to the dose of exergaming volume and that this effect weakened with additional 8 weeks of detraining (Fig. 2c). That is, regardless of exergaming $1 \times, 2 \times$, or $3 \times 1$ week, the greater the benefits of exergaming were, the better these gains were preserved. The pattern of lasting effects shown in Figure 2 was evident in the secondary outcomes (detailed correlation data not shown).

Withdrawal of the training stimulus with respect to balance produced highly inconsistent results. In mobility-limited Parkinsonian patients, the mobility benefits produced by exergaming used here lasted up to 6 months [15]. In some studies $[8,9]$, but not in other studies [10-12], older adults were able to maintain the strength and balance training-induced functional gains. Because of the high intensity of the sensorimotor stimulus during training, balance in particular, assessed here by fast gait speed, BBS, SPPB, and beam walking, can perhaps be selectively resistant to detraining, agreeing with previously proposed hypotheses [36]. We also observed a robust resistance to detraining for 16 weeks especially in G2 in exergaming-induced increases in fitness through heart rate responses to a standard step test, agreeing with some $[11,12,37]$ but not all cardiovascular detraining data [38-41]. The highly inconsistent de-

Gerontology 2021;67:403-414 411 


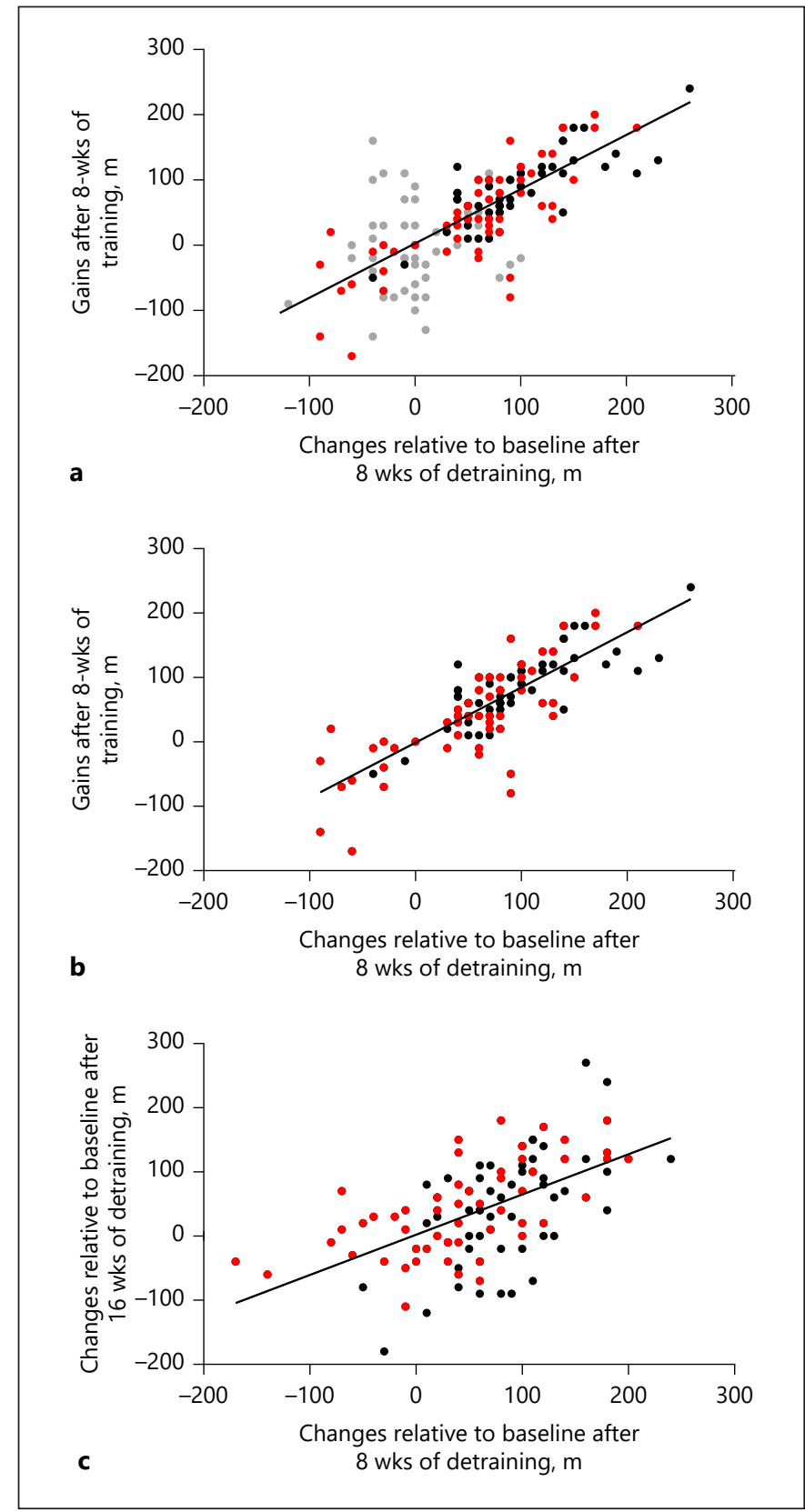

Fig. 2. Relationship between 8 weeks of exercise training-induced increases in the distance walked in $6 \mathrm{~min}$ and changes relative to baseline in distance walked in 6 min after 8 weeks of detraining in individual participants who exercised $1 \times$ (gray, $n=53$ ), $2 \times$ (black, $n=53$ ), or $3 \times$ (red, $n=54)$ per week (a) and in individual participants who only exercised $2 \times$ (black) or $3 \times$ (red) per week (b) and were retested after an additional 8 weeks of detraining, a total of 16 weeks of detraining (c). The figures show that those who gained the most after training maintained most of those gains in distance walked in 6 min after detraining. The maintenance of training gains after detraining is independent of training volume and weakens with prolonged detraining (c). The relationships are characterized by $y=0.79 x+1.1, R^{2}=0.52(\mathbf{a}) ; y=0.86 x-1.3, R^{2}=0.64(\mathbf{b})$; and $y=0.63 x+2.0, R^{2}=0.31$ (all $\beta$ coefficients, $R^{2}$ values $\left.p<0.001\right)(\mathbf{c})$. training effects on cardiovascular function might be related to differences between studies in using test intensity (maximal and submaximal), evaluation methods, participants' age, and training intensity, volume, and duration.

Training improved DSST and Stroop word-color time only (Table 1). These effects were sustained only in the Stroop test for 8 but not for 16 weeks of detraining, confirming to the dose effect we observed for motor outcomes (Tables 2,3). These data differ from the only detraining study we are aware of reporting a complete reversal of timed-up-and-go dual task performance to baseline after just 1 month of detraining in chronic strength-trained older women [42]. Exercise cessation reduced exercise-improved quality of life $[11,43]$ and health [44], but training and detraining did not affect blood-derived neurotrophic factors in healthy older adults [45]. Taking the current detraining data together, we found evidence that instead of the dose of training volume, training-induced gains per se determined the lasting exergaming effects on motor and only on one cognitive function in adults at late midlife.

\section{Limitations}

One limitation is the short intervention duration. However, when normalized for the number of sessions, outcome gains in longer exergaming studies are often similar to the gains reported in studies as short as the present work, implying a ceiling in the responses to the exercise stimulus [12]. Without a maintenance program, we cannot tell if the intervention-induced gains in mobility could be maintained and slow progression of mobility limitation. The small sample size prevented us to perform sex-stratified analyses. PA was measured by IPAQ instead of a wearable device. The substantial, $\sim 3.2 \mathrm{~kg}$, reduction in body mass implies that participants might have modified their diet, which we did not monitor. While the $100 \%$ adherence and $0 \%$ dropout suggest that our participants tolerated well the high exercise intensity, specially trained therapists delivered exercise sessions in a designated hospital facility, conditions unavailable elsewhere. However, participants could follow recent trends and perform agility exercises at home with remote supervision, reducing costs and staff burden. Without neural, physiological, or biomechanical markers, we were unable to determine the mechanisms of adaptations to training and detraining.

\section{Perspective}

Office workers near retirement tend to be sedentary and prone to mobility limitations and illnesses. Because exercise and PA recommendations are universal for broad age 
categories without considerations for current level of mobility, it is unclear how much exercise could ameliorate the ill effects of sedentariness and reduce mobility limitations at late midlife. How long exercise effects last is another contentious issue, considering the wide range of findings of no residual effects at all and effects that outlasted the training stimulus for several months. The current results inform physicians, therapists, and fitness specialists that less training could be more, with mobility and cognitive improvements lasting for up to 16 weeks after the end of the exercise program in adults at late midlife with mild mobility limitations at baseline. The data expand current guidelines of exercise prescription by showing that twice weekly enjoyable but vigorous exergaming can produce lasting effects on mobility and cognition in initially sedentary adults aged 60 with mild mobility limitations.

\section{Conclusion}

We found that less was more during training and lasted longer after detraining: medium duration dose of exergaming produced the largest clinically meaningful improvements in mobility and selected cognitive tests in 60-year-old office workers with mild mobility limitations and intact cognition.

\section{Acknowledgements}

This study was supported in part by the Department of Neurology, Somogy County Móricz Kaposi Teaching Hospital, and supported by the UNNP-20-4-II, New National Excellence Program of the Ministry for Innovation and Technology from the source of the National Research, Development and Innovation Fund. We thank the participants for their time and effective contribution to the study.

\section{Statement of Ethics}

Each participant provided written informed consent for the study, which was conducted in compliance with the norms of the Declaration of Helsinki (2013 version). The study was approved by the Ethics Committee at the Somogy County Móricz Kaposi Teaching Hospital, Kaposvár, Hungary(Ethics No.IKEB009/2018). Clinical trial: NCT03743363.

\section{Conflict of Interest Statement}

The authors declare that they have no conflicts of interest.

\section{Author Contributions}

T.H., J.T., and U.G. conceived, designed, and contributed to the data collection. T.H., J.T., and U.G. collected and analyzed the data. All authors contributed to the interpretation of the data and to the preparation and editing of the manuscript.

\section{References}

1 World Health Organization. Global action plan on physical activity 2018-2030: more active people for a healthier world. Geneva: World Health Organization; 2018.

2 Prince SA, Elliott CG, Scott K, Visintini S, Reed JL. Device-measured physical activity, sedentary behaviour and cardiometabolic health and fitness across occupational groups: a systematic review and meta-analysis. Int $\mathrm{J}$ Behav Nutr Phys Act. 2019 Apr 2;16(1):30.

3 Arem H, Moore SC, Patel A, Hartge P, Berrington de Gonzalez A, Visvanathan $\mathrm{K}$, et al. Leisure time physical activity and mortality: a detailed pooled analysis of the dose-response relationship. JAMA Intern Med. 2015 Jun; 175(6):959-67.

4 Piercy KL, Troiano RP, Ballard RM, Carlson SA, Fulton JE, Galuska DA, et al. The physical activity guidelines for Americans. JAMA. 2018 Nov 20;320(19):2020-8.

5 Benestad HB, Sand KL, Bruusgaard JC. Less than recommended training of aerobic fitness and muscle strength: What to expect? Acta Physiol. 2018;224(4):1-2.
6 Moreno-Agostino D, Daskalopoulou C, Wu YT, Koukounari A, Haro JM, Tyrovolas S, et al. The impact of physical activity on healthy ageing trajectories: evidence from eight cohort studies. Int J Behav Nutr Phys Act. 2020 Jul 16;17(1):92.

7 Brahms CM, Hortobagyi T, Kressig RW, Granacher $\mathrm{U}$. The interaction between mobility status and exercise specificity in older adults. Exerc Sport Sci Rev. 2021 Jan;49(1):15-22.

8 Van Roie E, Walker S, Van Driessche S, Baggen R, Coudyzer W, Bautmans I, et al. Training load does not affect detraining's effect on muscle volume, muscle strength and functional capacity among older adults. Exp Gerontol. 2017 Nov;98:30-7.

9 Orange ST, Marshall P, Madden LA, Vince RV. Short-term training and detraining effects of supervised vs. unsupervised resistance exercise in aging adults. J Strength Cond Res. 2019 Oct;33(10):2733-42.

10 Coetsee C, Terblanche E. The time course of changes induced by resistance training and detraining on muscular and physical function in older adults. Eur Rev Aging Phys Act. 2015; 12:7.
11 Esain I, Gil SM, Bidaurrazaga-Letona I, Rodriguez-Larrad A. Effects of 3 months of detraining on functional fitness and quality of life in older adults who regularly exercise. Aging Clin Exp Res. 2019 Apr;31(4):503-10.

12 Villafaina S, Borrega-Mouquinho Y, FuentesGarcía JP, Collado-Mateo D, Gusi N. Effect of exergame training and detraining on lowerbody strength, agility, and cardiorespiratory fitness in women with fibromyalgia: singleblinded randomized controlled trial. Int J Environ Res Public Health. 2019 Dec 24;17(1): 161.

13 Stanmore E, Stubbs B, Vancampfort D, de Bruin ED, Firth J. The effect of active video games on cognitive functioning in clinical and non-clinical populations: a meta-analysis of randomized controlled trials. Neurosci Biobehav Rev. 2017 Jul;78:34-43.

14 Corregidor-Sanchez AI, Segura-Fragoso A, Rodriguez-Hernandez M, Criado-Alvarez JJ, Gonzalez-Gonzalez J, Polonio-Lopez B. Can exergames contribute to improving walking capacity in older adults? A systematic review and meta-analysis. Maturitas. 2020 Feb;132: $40-8$. 
15 Tollar J, Nagy F, Kovacs N, Hortobagyi T. Two-year agility maintenance training slows the progression of Parkinsonian symptoms. Med Sci Sports Exerc. 2019 Feb;51(2):237-45.

16 Tollar J, Nagy F, Kovacs N, Hortobagyi T. A high-intensity multicomponent agility intervention improves Parkinson patients' clinical and motor symptoms. Arch Phys Med Rehabil. 2018 Dec;99(12):2478-84.e1.

17 Tollar J, Nagy F, Moizs M, Toth BE, Sanders LMJ, Hortobagyi T. Diverse exercises similarly reduce older adults' mobility limitations. Med Sci Sports Exerc. 2019 Sep;51(9):180916.

18 Tollar J, Nagy F, Toth BE, Torok K, Szita K, Csutoras B, et al. Exercise effects on multiple sclerosis quality of life and clinical-motor symptoms. Med Sci Sports Exerc. 2020 May; 52(5):1007-14.

19 Bean JF, Kiely DK, Leveille SG, Herman S, Huynh C, Fielding R, et al. The 6-minute walk test in mobility-limited elders: What is being measured? J Gerontol A Biol Sci Med Sci. 2002 Nov;57(11):M751-6.

20 Perera S, Mody SH, Woodman RC, Studenski SA. Meaningful change and responsiveness in common physical performance measures in older adults. J Am Geriatr Soc. 2006 May; 54(5):743-9.

21 Hortobágyi T, Lesinski M, Gäbler M, VanSwearingen JM, Malatesta D, Granacher U. Effects of three types of exercise interventions on healthy old adults' gait speed: a systematic review and meta-analysis. Sports Med. 2015; 45:1627-43.

22 Guralnik JM, Simonsick EM, Ferrucci L, Glynn RJ, Berkman LF, Blazer DG, et al. A short physical performance battery assessing lower extremity function: association with self-reported disability and prediction of mortality and nursing home admission. J Gerontol. 1994 Mar;49(2):M85-94.

23 Berg KO, Maki BE, Williams JI, Holliday PJ, Wood-Dauphinee SL. Clinical and laboratory measures of postural balance in an elderly population. Arch Phys Med Rehabil. 1992 Nov;73(11):1073-80.

24 Hortobágyi T, Uematsu A, Sanders L, Kliegl $\mathrm{R}$, Tollár J, Moraes R, et al. Beam walking to assess dynamic balance in health and disease: a protocol for the "BEAM" multicenter observational study. Gerontology. 2019;65(4):3329.
25 Shephard DA. Editorial: home testing of fitness of Canadians. Can Med Assoc J. 1976 Apr 17;114(8):662-79.

26 Smith L, Hamer M, Ucci M, Marmot A, Gardner B, Sawyer A, et al. Weekday and weekend patterns of objectively measured sitting, standing, and stepping in a sample of officebased workers: the active buildings study. BMC Public Health. 2015 Jan 17;15:9.

27 Lee PH, Macfarlane DJ, Lam TH, Stewart SM. Validity of the International Physical Activity Questionnaire Short Form (IPAQ-SF): a systematic review. Int J Behav Nutr Phys Act. 2011 Oct 21;8:115.

28 Seo YG, Noh HM, Kim SY. Weight loss effects of circuit training interventions: a systematic review and meta-analysis. Obes Rev. 2019 Nov;20(11):1642-50.

29 Fielding RA, Travison TG, Kirn DR, Koochek A, Reid KF, von Berens Å, et al. Effect of structured physical activity and nutritional supplementation on physical function in mobilitylimited older adults: results from the VIVE2 randomized trial. J Nutr Health Aging. 2017; 21(9):936-42.

30 Lesinski M, Hortobágyi T, Muehlbauer T, Gollhofer A, Granacher U. Effects of balance training on balance performance in healthy older adults: a systematic review and meta-analysis. Sports Med. 2015 Dec;45(12):1721-38.

31 Boter H, Mänty M, Hansen AM, Hortobágyi $\mathrm{T}$, Avlund K. Self-reported fatigue and physical function in late mid-life. J Rehabil Med. 2014 Jun 25;46(7):684-90.

32 Clouston SA, Brewster P, Kuh D, Richards M, Cooper R, Hardy R, et al. The dynamic relationship between physical function and cognition in longitudinal aging cohorts. Epidemiol Rev. 2013;35:33-50.

33 Best JR, Liu-Ambrose T, Boudreau RM, Ayonayon HN, Satterfield S, Simonsick EM, et al. An evaluation of the longitudinal, bidirectional associations between gait speed and cognition in older women and men. J Gerontol A Biol Sci Med Sci. 2016 Dec;71(12):1616-23.

34 Kikkert LHJ, Vuillerme N, van Campen JP, Hortobágyi T, Lamoth CJ. Walking ability to predict future cognitive decline in old adults: a scoping review. Ageing Res Rev. 2016 May; 27:1-14.

35 Morris R, Lord S, Bunce J, Burn D, Rochester L. Gait and cognition: mapping the global and discrete relationships in ageing and neurodegenerative disease. Neurosci Biobehav Rev. 2016 May;64:326-45.
36 Carvalho MJ, Marques E, Mota J. Training and detraining effects on functional fitness after a multicomponent training in older women. Gerontology. 2009;55(1):41-8.

37 Ratel S, Gryson C, Rance M, Penando S, Bonhomme C, Le Ruyet P, et al. Detraining-induced alterations in metabolic and fitness markers after a multicomponent exercisetraining program in older men. Appl Physiol Nutr Metab. 2012 Feb;37(1):72-9.

38 Toraman NF. Short term and long term detraining: Is there any difference between young-old and old people? Br J Sports Med. 2005 Aug;39(8):561-4.

39 Bocalini DS, Serra AJ, Rica RL, Dos Santos L. Repercussions of training and detraining by water-based exercise on functional fitness and quality of life: a short-term follow-up in healthy older women. Clinics. 2010;65(12): 1305-9.

40 St-Amand J, Yoshioka M, Nishida Y, Tobina T, Shono N, Tanaka H. Effects of mild-exercise training cessation in human skeletal muscle. Eur J Appl Physiol. 2012 Mar;112(3):85369.

41 Murias JM, Edwards JA, Paterson DH. Effects of short-term training and detraining on $\mathrm{VO} 2$ kinetics: faster $\mathrm{VO} 2$ kinetics response after one training session. Scand J Med Sci Sports. 2016 Jun;26(6):620-9.

42 Coelho HJ, Rodrigues B, de Oliveira Gonçalves I, Uchida MC. Effects of a short-term detraining period on muscle functionality and cognition of strength-trained older women: a preliminary report. J Exerc Rehabil. 2017 Oct;13(5):559-67.

43 Lobo A, Carvalho J, Santos P. Effects of training and detraining on physical fitness, physical activity patterns, cardiovascular variables, and HRQoL after 3 health-promotion interventions in institutionalized elders. Int J Family Med. 2010;2010:486097.

44 Leitao L, Pereira A, Mazini M, Venturini G, Campos Y, Vieira J, et al. Effects of three months of detraining on the health profile of older women after a multicomponent exercise program. Int J Environ Res Public Health. 2019 Oct 13;16(20):3881.

45 Goekint M, Roelands B, De Pauw K, Knaepen K, Bos I, Meeusen R. Does a period of detraining cause a decrease in serum brain-derived neurotrophic factor? Neurosci Lett. 2010 Dec $17 ; 486(3): 146-9$. 